\title{
Synthesis of Cyclic and Acyclic Pyrimidine Nucleosides Analogues with Anticipated Antiviral Activity
}

\author{
Mohamed F. El-Shehry, ${ }^{1, *}$ Emad M. El Telbani ${ }^{2,3}$ \\ and Mohamed I. Hegab ${ }^{4,5}$ \\ ${ }^{1}$ Pesticides Chemistry Department, National Research Centre, Dokki, 12622 Giza, Egypt \\ ${ }^{2}$ Green Chemistry Department, National Research Centre, Dokki, 12622 Giza, Egypt \\ ${ }^{3}$ Chemistry Department, Faculty of Science, Jazan University, Jazan, Saudi Arabia \\ ${ }^{4}$ Photochemistry Department, National Research Centre, Dokki, 12622 Giza, Egypt \\ ${ }^{5}$ Chemistry Department, Faculty of Science \& Arts, Qurayat, Al-Jouf University, Saudi Arabia \\ *Corresponding author: E-mail:moh_elshehry2000@yahoo.com
}

Received: 25-12-2017

\begin{abstract}
A convenient method for preparation of cyclic and acyclic nucleosides was achieved by alkylation of 6-(2,4-dichlorophenoxymethyl)pyrimidine-2,4-dione (1) with a variety of acyclic and cyclic activated sugar analogues, namely (2-acetoxyethoxy)methyl acetate (3), 2-(acetoxymethoxy)propane-1,3-diyl dibenzoate (4), benzyloxymethyl acetate (5), 2-acetoxy-5-(benzoyloxymethyl)tetrahydrofuran-3,4-diyl dibenzoate (12), 5-chloro-2-((4-chlorobenzoyloxy)methyl) tetrahydrofuran-3-yl 4-chlorobenzoate (13) and 2-(acetoxymethyl)-6-bromotetrahydro-2H-pyran-3,4,5-triyl triacetate (14), respectively. Deprotection of the synthesized nucleosides was achieved by using methanolic ammonia. The structures of the newly synthesized nucleoside analogues were fully characterized by analytical methods (mass spectrometry, ${ }^{1} \mathrm{H}$ NMR, ${ }^{13} \mathrm{C}$ NMR, and elemental analysis).
\end{abstract}

Keywords: Pyrimidines, nucleosides, vorbrüggen and niedballa's procedure, antiviral activity

\section{Introduction}

A number of base-modified nucleosides have been playing a vital and important role as therapeutic agents in the treatment of patients infected with different viruses including human immunodeficiency virus (HIV), herpes simplex virus (HSV), hepatitis B virus (HBV), hepatitis C virus (HCV) and cytomegalovirus (CMV) infections. ${ }^{1}$ According to U.S. Food and Drug Administration (FDA), many cyclic and acyclic nucleoside analogues, such as 3'-azido-3'-deoxythymidine (AZT), 2',3'-dideoxyinosidine (DDI), 2',3'-didehydro-3'-deoxythymidine (D4T), 1-[(2-hydroxyethoxy)methyl]-6-(phenylthio)thymine (HEPT), acyclovir, and penciclovir (Fig. 1) are effective in treatment of various viruses. ${ }^{2}$

Moreover, it is well known that functionalized nitrogen heterocycles play an interesting role in drug chemistry and therefore they have been intensively studied and used as scaffolds for searching and developing new drugs. ${ }^{3}$ Py- rimidine-incorporating sugar residues represent an interesting class of nucleosides which have a promising antiviral chemotherapy potential, especially that class in which the cyclic sugar residue is replaced with open-chain "acyclic" sugar moieties. Moreover, heterocycles possessing pyrimidine nucleus are of great interest because they constitute an important class of natural and non-natural products which possess diverse biological activities and medicinal applications. ${ }^{4}$ Additionally, pyrimidine skeleton is also present in many natural products, such as vitamin B1 (thiamine) and a lot of synthetic compounds which possess a wide spectrum of biological activities including polio herpes viruses,${ }^{5}$ diuretic, anti-HIV, cardiovascular, ${ }^{6}$ antibacterial, ${ }^{7-9}$ antifungal, ${ }^{10,11}$ antihypertensive, ${ }^{12}$ antipyretic, ${ }^{13}$ antiviral, ${ }^{14-15}$ antidiabetic, ${ }^{16}$ antioxidant, ${ }^{17-18}$ anticancer activities, ${ }^{19-20}$ antileishmanial, ${ }^{21}$ anti-inflammatory, ${ }^{22}$ analgesic, ${ }^{23}$ antiallergic, ${ }^{24}$ anticonvulsant, ${ }^{25}$ antihistaminic, ${ }^{26}$ herbicidal, ${ }^{27}$ antidepressant, ${ }^{28}$ and also act as calcium 


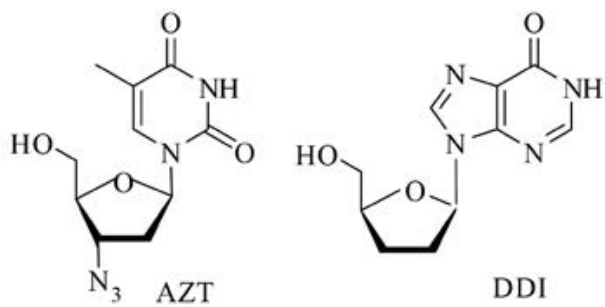<smiles>Cc1cn([C@@H]2C=C[C@@H](CO)O2)c(=O)[nH]c1=O</smiles>

D4T<smiles>Cc1c(Sc2ccccc2)n(COCCO)c(=O)[nH]c1=O</smiles>

HEPT<smiles>Nc1nc2c(ncn2COCCO)c(=O)[nH]1</smiles><smiles>Nc1nc2c(ncn2CCC(CO)CO)c(=O)[nH]1</smiles>

Figure 1: Some examples of cyclic and acyclic antiviral agents

channel blockers. ${ }^{29}$ On the other side, fusion of pyrimidine moiety with different heterocycle scaffolds gives rise to a new class of hybrid heterocycles possessing improved biological activity. Fused pyrimidines like purines, quinazolines, pteridines, pyridopyrimidines, pyrazolopyrimidines, pyrimidoazepines, triazolopyrimidines, furopyrimidines and pyrrolopyrimidines were studied in the past decade and were found to possess remarkable pharmacological properties, such as antibacterial activity, antifungal activity, anti-cancer agents, antihyperlipidemic activity, blood related disorders, analgesic and anti-inflammatory activities, anti-HIV agents, CNS related agents, and immunosuppressants. ${ }^{30}$

For the design and search of new drugs, the development of hybrid molecules through the binding of various pharmacophores in one frame could lead to molecules with interesting pharmaceutical properties. Based on above information and in continuations of our interest in the synthesis of bioactive molecules derived from the pyrimidine moeity, ${ }^{15,31-33}$ our investigation aimed to synthesize new cyclic and acyclic nucleosides using available cyclic and acyclic moieties, which will be coupled with pyrimidine base, hoping to increase their antiviral activities. The effective method of protection and deprotection will also be examined.

\section{Experimental}

\section{1. Chemistry}

All the reagents were purchased from Sigma-Aldrich and the solvents from Merck and were used without further purification. Melting points were measured on an Apotec apparatus and are uncorrected. NMR spectra were recorded on Bruker AMX400 and Bruker Current AV400 Data spectrometers $\left(400 \mathrm{MHz}\right.$ for ${ }^{1} \mathrm{H}, 100.6 \mathrm{MHz}$ for ${ }^{13} \mathrm{C}$ ). ESI mass spectra were determined with a Finnigan Thermo Quest MAT 95XL spectrometer and FAB high-resolu- tion (HR) mass spectra with a VG Analytical 70-250S spectrometer using an MCA method and poly(ethylene glycol) as the support. The reactions were monitored by thin layer chromatography (TLC) using silica gel (60 F254) coated aluminium plates (Merck) which were visualized by UV irradiation $(254 \mathrm{~nm})$ and iodine vapours. Column chromatography was performed by using silica gel (60120 mesh). All reactions were carried out under dry nitrogen. 6-(2,4-Dichlorophenoxymethyl)pyrimidine-2,4-dione (1) was prepared according to our previous report. ${ }^{15}$

\section{1. 1. Preparation of Nucleosides $6,8,10,11,16$, 15,17 and 19}

A suspension of uracil derivative $\mathbf{1}(10 \mathrm{mmol})$ and ammonium sulphate $(10 \mathrm{~g})$ in HMDS $(50 \mathrm{~mL})$ was stirred and refluxed for $4 \mathrm{~h}$. HMDS in excess was evaporated under reduced pressure to give bis(trimethylsilyl) compound 2 . A solution of acylated acyclic reagents $(10 \mathrm{mmol})$ : $(2$-acetoxyethoxy)methyl acetate (3), 2-(acetoxymethoxy)propane-1,3-diyl dibenzoate (4), benzyloxymethyl acetate (5), 2-acetoxy-5-(benzoyloxymethyl)tetrahydrofuran-3,4-diyl dibenzoate (12), 5-chloro-2-((4-chlorobenzoyloxy)methyl) tetrahydrofuran-3-yl 4-chlorobenzoate (13) and 2-(acetoxymethyl)-6-bromotetrahydro- $2 \mathrm{H}$-pyran-3,4,5-triyl triacetate (14), in dry acetonitrile $(30 \mathrm{~mL})$ and tin(IV) chloride $(2 \mathrm{~mL})$ was individually added to the residue of 2 and stirred at $-30^{\circ} \mathrm{C}$ for $24 \mathrm{~h}$. After addition of pyridine $(4 \mathrm{~mL})$ the mixture was filtered to remove inorganic materials. The filtrate was diluted with chloroform $(40 \mathrm{~mL})$. The organic layer was washed with a saturated solution of sodium hydrogen carbonate $(50 \mathrm{~mL})$, followed by a $1 \mathrm{~N}$ solution of hydrochloric acid $(50 \mathrm{~mL})$, then brine $(50 \mathrm{~mL})$ and water successively, dried over anhydrous sodium sulfate and concentrated to dryness under reduced pressure. The resulting crude nucleosides 6, 8, 10, 11, 15, 17 and 19 were separated by silica gel column chromatography (graduated mixture of ethyl acetate and pethroleum ether, 9:1) as white solid. 
2-((6-((2,4-Dichlorophenoxy) methyl)-2,4-dioxo-3,4-dihydropyrimidin-1(2H)-yl)methoxy)ethyl Acetate (6) Yield $2.8 \mathrm{~g}$ (70\%), m.p. $166-168{ }^{\circ} \mathrm{C} .{ }^{1} \mathrm{H}$ NMR (DMSO- $d_{6}$, $400 \mathrm{MHz}) \delta 2.11\left(\mathrm{~s}, 3 \mathrm{H}, \mathrm{COCH}_{3}\right), 3.83,4.42(\mathrm{t}, 4 \mathrm{H}, \mathrm{OCH}-$ $\left.{ }_{2} \mathrm{CH}_{2} \mathrm{O}\right), 5.45\left(\mathrm{~s}, 2 \mathrm{H}, \mathrm{CH}_{2}\right.$ phenoxy), $5.62\left(\mathrm{~s}, 2 \mathrm{H}, \mathrm{OCH}_{2} \mathrm{~N}\right)$, 6.10 (s, 1H, CH uracil), 7.48-7.90 (m, 3H, Ar-H), 12.50 (br s, $1 \mathrm{H}, \mathrm{NH}) .{ }^{13} \mathrm{C}$ NMR (DMSO- $\left.d_{6}, 100 \mathrm{MHz}\right) \delta 20.9,63.2$, $65.1,66.8,72.1,100.7,115.9,116.0,122.9,126.1,128.6$, $129.9,151.5,152.1,162.7,170.6$. MS $m / z=402$ [M-1]. Anal. Calcd for $\mathrm{C}_{16} \mathrm{H}_{16} \mathrm{Cl}_{2} \mathrm{~N}_{2} \mathrm{O}_{6}$ : C, 47.66; $\mathrm{H}, 4.00 ; \mathrm{N}, 6.95$. Found: C, 47.81; H, 4.09; N, 6.79.

2-((6-((2,4-Dichlorophenoxy) methyl)-2,4-dioxo-3,4-dihydropyrimidin-1(2H)-yl)methoxy)propane-1,3-diyl Dibenzoate (8)

Yield $4.3 \mathrm{~g}$ (73\%), m.p. $162-164{ }^{\circ} \mathrm{C} .{ }^{1} \mathrm{H}$ NMR (DMSO- $d_{6}$, $400 \mathrm{MHz}) \delta 4.20-4.50\left(\mathrm{~m}, 5 \mathrm{H}, 2 \mathrm{CH}_{2}, \mathrm{CH}\right), 5.11(\mathrm{~s}, 2 \mathrm{H}$, $\mathrm{CH}_{2}$ phenoxy), $5.41\left(\mathrm{~s}, 2 \mathrm{H}, \mathrm{OCH}_{2} \mathrm{~N}\right), 5.62(\mathrm{~s}, 1 \mathrm{H}, \mathrm{CH}$ uracil), 7.10-7.93 (m, 13H, Ar-H), 12.10 (br s, $1 \mathrm{H}, \mathrm{NH}) .{ }^{13} \mathrm{C}$ NMR (DMSO- $\left.d_{6}, 100 \mathrm{MHz}\right) \delta 64.1,64.8,71.3,74.3,100.6$, $115.6,122.8,125.9,128.4,129.0,129.5,129.8,133.8,151.3$, 151.7, 152.2, 162.5, 165.8. MS $m / z=599.2\left[\mathrm{M}^{+}\right]$. Anal. Calcd for $\mathrm{C}_{29} \mathrm{H}_{24} \mathrm{Cl}_{2} \mathrm{~N}_{2} \mathrm{O}_{8}$ : C, 58.11; H, 4.04; N, 4.67. Found: C, 58.24; H, 4.13; N, 4.53 .

1-(Benzyloxymethyl)-6-((2,4-dichlorophenoxy)methyl) pyrimidine-2,4(1H,3H)-dione (10)

Yield $2.8 \mathrm{~g}$ (69\%), m.p. $114-116{ }^{\circ} \mathrm{C} .{ }^{1} \mathrm{H}$ NMR (DMSO- $d_{6}$, $400 \mathrm{MHz}$ ): $\delta 4.62\left(\mathrm{~s}, 2 \mathrm{H}, \mathrm{CH}_{2} \mathrm{Ph}\right), 5.25$ (s, $2 \mathrm{H}, \mathrm{CH}_{2}$ phenoxy), 5.45 (s, $\left.2 \mathrm{H}, \mathrm{OCH}_{2} \mathrm{~N}\right), 5.81$ (s, $1 \mathrm{H}, \mathrm{CH}$ uracil), 7.287.81 (m, 8H, Ar-H), 12.01 (br s, $1 \mathrm{H}, \mathrm{NH}) .{ }^{13} \mathrm{C}$ NMR (DMSO- $\left.d_{6}, 100 \mathrm{MHz}\right): \delta 64.8,70.3,71.5,100.6,115.6,122.6$, 125.7, 127.0, 127.7, 128.1, 128.2, 129.5, 137.3, 150.9, 151.5, 151.6, 158.8. MS: $m / z=407.2\left[\mathrm{M}^{+}\right]$. Anal. Calcd for $\mathrm{C}_{19} \mathrm{H}-$ ${ }_{16} \mathrm{Cl}_{2} \mathrm{~N}_{2} \mathrm{O}_{4}: \mathrm{C}, 56.04 ; \mathrm{H}, 3.96 ; \mathrm{N}, 6.88$. Found: C, 56.11; $\mathrm{H}$, 3.87; N, 6.97.

1,3-Bis(benzyloxymethyl)-6-((2,4-dichlorophenoxy) methyl)pyrimidine-2,4(1H,3H)-dione (11)

Yield $3 \mathrm{~g}$ (58\%), m.p. $158-160{ }^{\circ} \mathrm{C} .{ }^{1} \mathrm{H}$ NMR (DMSO- $d_{6}$, $400 \mathrm{MHz}) \delta 4.43,4.45\left(2 \times \mathrm{s}, 4 \mathrm{H}, 2 \times \mathrm{CH}_{2} \mathrm{Ph}\right), 5.24(\mathrm{~s}, 2 \mathrm{H}$, $\mathrm{CH}_{2}$ phenoxy), $5.34\left(\mathrm{~s}, 2 \mathrm{H}, \mathrm{OCH}_{2}-\mathrm{N}^{1}\right), 5.46(\mathrm{~s}, 2 \mathrm{H}$, $\left.\mathrm{OCH}_{2}-\mathrm{N}^{3}\right), 5.91(\mathrm{~s}, 1 \mathrm{H}, \mathrm{CH}$ uracil), 7.25-7.67 (m, 13H, Ar-H). ${ }^{13} \mathrm{C}$ NMR (DMSO- $\left.d_{6}, 100 \mathrm{MHz}\right) \delta 65.1,70.6,71.4$, $73.1,100.2,116.0,122.9,126.1,127.7,127.8,128.1,128.5$, $128.6,129.9,137.7,150.8,152.2,161.6$. MS $m / z=527.3$ $\left[\mathrm{M}^{+}\right]$. Anal. Calcd for $\mathrm{C}_{27} \mathrm{H}_{24} \mathrm{Cl}_{2} \mathrm{~N}_{2} \mathrm{O}_{5}: \mathrm{C}, 61.49 ; \mathrm{H}, 4.59 ; \mathrm{N}$, 5.31. Found: C, 61.55; H, 4.51; N, 5.39.

2-(Benzoyloxymethyl)-5-(6-((2,4-dichlorophenoxy) methyl)-2,4-dioxo-3,4-dihydropyrimidin-1(2H)-yl)tetrahydrofuran-3,4-diyl Dibenzoate (15)

Yield $4.5 \mathrm{~g}(62 \%)$, m.p. $113-115^{\circ} \mathrm{C} .{ }^{1} \mathrm{H}$ NMR (DMSO- $d_{6}$, $400 \mathrm{MHz}) \delta 4.31-4.40$ (m, 2H, H-5,5"), 4.51-4.60 (m, 1H, $\left.\mathrm{H}-4^{\prime}\right), 4.91$ (s, $2 \mathrm{H}, \mathrm{CH}_{2}$ phenoxy), $5.71(\mathrm{~s}, 1 \mathrm{H}, \mathrm{CH}$ uracil), 5.90-6.11 (m, 2H, H-2', H-3'), $6.40(\mathrm{~d}, 1 \mathrm{H}, J=9.10 \mathrm{~Hz}$,
H-1'), 7.10-7.98 (m, 18H, Ar-H), 12.01 (br s, $1 \mathrm{H}, \mathrm{NH}) .{ }^{13} \mathrm{C}$ NMR (DMSO- $\left.d_{6}, 100 \mathrm{MHz}\right) \delta 63.7,65.7,70.7,73.8,78.2$, 98.0, 115.7, 122.9, 125.9, 128.6, 128.8, 128.9, 129.1, 129.5, $129.6,129.9,133.7,134.0,134.2,151.1,152.2,162.5,164.9$, 165.1, 165.8. MS $m / z=730[\mathrm{M}-1]$. Anal. Calcd for $\mathrm{C}_{37} \mathrm{H}-$ ${ }_{28} \mathrm{Cl}_{2} \mathrm{~N}_{2} \mathrm{O}_{10}$ : C, 60.75; H, 3.86; N, 3.83. Found: C, 60.63; H, $3.94 ; \mathrm{N}, 3.75$.

(3-(4-Chlorobenzoyloxy)-5-(6-((2,4-dichlorophenoxy) methyl)-2,4-dioxo-3,4-dihydropyrimidin-1(2H)-yl)tetrahydrofuran-2-yl)methyl 4-Chlorobenzoate (17)

Yield 3.9 g (58\%), m.p. $85-87^{\circ} \mathrm{C} .{ }^{1} \mathrm{H}$ NMR (DMSO- $d_{6}, 400$ $\mathrm{MHz}) \delta 2.21-2.30(\mathrm{~m}, 2 \mathrm{H}, \mathrm{H}-2,2$ '), $4.81-4.25$ (m, $2 \mathrm{H}$, H-5,5”), 4.72 (m, 1H, H-4'), 4.90 (s, $2 \mathrm{H}, \mathrm{CH}_{2}$ phenoxy), 5.40 (m, 1H, H-3'), $5.61\left(\mathrm{~s}, 1 \mathrm{H}, \mathrm{CH}\right.$ uracil), $6.51\left(\mathrm{~m}, 1 \mathrm{H}, \mathrm{H}-\mathrm{l}^{\prime}\right)$, 7.10-8.00 (m, 11H, Ar-H), 11.93 (br s, $1 \mathrm{H}, \mathrm{NH}) .{ }^{13} \mathrm{C}$ NMR $\left(\mathrm{DMSO}-d_{6}, 100 \mathrm{MHz}\right) \delta 66.4,67.6,74.9,75.6,79.5,81.2$, $82.3,98.2,102.9,115.6,128.5,129.2,129.8,129.9,130.6$, $131.1,131.4,138.6,150.4,150.8,151.1,162.8,163.0,164.9$. MS $m / z=678$ [M-2]. Anal. Calcd for $\mathrm{C}_{30} \mathrm{H}_{22} \mathrm{Cl}_{4} \mathrm{~N}_{2} \mathrm{O}_{8}$ : C, 52.96; H, 3.26; N, 4.12. Found: C, 52.87; H, 3.31; N, 4.20.

2-(Acetoxymethyl)-6-(6-((2,4-dichlorophenoxy)methyl)-2,4-dioxo-3,4-dihydropyrimidin-1(2H)-yl)tetrahydro-2H-pyran-3,4,5-triyl Triacetate (19)

Yield $4.1 \mathrm{~g}(67 \%)$, m.p. $137-139{ }^{\circ} \mathrm{C} .{ }^{1} \mathrm{H}$ NMR (DMSO- $d_{6}$, $400 \mathrm{MHz}) \delta 1.80-2.10\left(4 \times \mathrm{s}, 12 \mathrm{H}, 4 \times \mathrm{COCH}_{3}\right), 3.31(\mathrm{~m}$, $\left.1 \mathrm{H}, \mathrm{H}-5^{\prime}\right), 4.18\left(\mathrm{~m}, 2 \mathrm{H}, \mathrm{H}-6^{\prime}, 6^{\prime \prime}\right), 5.02\left(\mathrm{~m}, 4 \mathrm{H}, \mathrm{H}-3^{\prime}\right.$, $\mathrm{H}-4, \mathrm{CH}_{2}$ phenoxy), $5.42\left(\mathrm{dd}, 1 \mathrm{H}, J_{1,2}=9.50, J_{2,3}=9.10 \mathrm{~Hz}\right.$, H-2'), $5.60\left(\mathrm{~s}, 1 \mathrm{H}, \mathrm{CH}\right.$ uracil), $6.20\left(\mathrm{~d}, 1 \mathrm{H}, J_{1,2}=9.50 \mathrm{~Hz}\right.$, $\mathrm{H}-1$ '), 7.10-7.81 (m, 3H, Ar-H), 12.01 (br s, $1 \mathrm{H}, \mathrm{NH}) .{ }^{13} \mathrm{C}$ NMR (DMSO- $\left.d_{6}, 100 \mathrm{MHz}\right) \delta 15.5,20.2,20.5,20.7,62.0$, $65.2,67.9,73.0,77.8,79.5,96.6,99.1,115.7,122.9,125.9$, $149.8,151.7,152.1,162.1,162.7,169.3,170.3 . \mathrm{MS} \mathrm{m} / \mathrm{z}=$ $617.3\left[\mathrm{M}^{+}\right]$. Anal. Calcd for $\mathrm{C}_{25} \mathrm{H}_{26} \mathrm{Cl}_{2} \mathrm{~N}_{2} \mathrm{O}_{12}$ : C, 48.64; $\mathrm{H}$, $4.24 ; \mathrm{N}, 4.54$. Found: C, 48.59; H, 4.31; N, 4.60.

\section{1. 2. General Procedure for the Preparation of Deprotected Nucleosides 7, 9, 16, 18 and 20}

Each protected nucleoside was dissolved individually in methanol saturated with ammonia and stirred for two days at room temperature. Then the solution was concentrated to dryness and the residue recrystallized in methanol to give deprotected nucleosides 7, 9, 16, 18 and 20.

6-((2,4-Dichlorophenoxy)methyl)-1-((2-hydroxyethoxy) methyl)pyrimidine-2,4(1H,3H)-dione (7)

Yield $3 \mathrm{~g}$ (83\%), m.p. $228-230^{\circ} \mathrm{C}$. ${ }^{1} \mathrm{H}$ NMR (DMSO- $d_{6}, 400$ $\mathrm{MHz}) \delta 3.70,3.76\left(2 \times \mathrm{t}, 4 \mathrm{H}, \mathrm{HOCH}_{2} \mathrm{CH}_{2} \mathrm{O}\right), 4.90(\mathrm{br} \mathrm{s}, 1 \mathrm{H}$, $\mathrm{OH}), 5.50\left(\mathrm{~s}, 2 \mathrm{H}, \mathrm{CH}_{2}\right.$ phenoxy), $5.56\left(\mathrm{~s}, 2 \mathrm{H}, \mathrm{OCH}_{2} \mathrm{~N}\right), 6.03$ (s, $1 \mathrm{H}, \mathrm{CH}$ uracil), $7.40-7.80$ (m, 3H, Ar-H), 11.83 (br s, $1 \mathrm{H}$, $\mathrm{NH}) .{ }^{13} \mathrm{C}$ NMR (DMSO- $\left.d_{6}, 100 \mathrm{MHz}\right) \delta 60.3,65.0,70.7$, 72.2, 100.5, 116.0, 122.9, 126.0, 128.6, 129.9, 151.9, 162.7. MS $m / z=360$ [M-1]. Anal. Calcd for $\mathrm{C}_{14} \mathrm{H}_{14} \mathrm{Cl}_{2} \mathrm{~N}_{2} \mathrm{O}_{5}$ : C, 46.56; H, 3.91; N, 7.76. Found: C, 46.62; H, 3.83; N, 7.84.

El-Shehry et al.: Synthesis of Cyclic and Acyclic Pyrimidine ... 
6-((2,4-Dichlorophenoxy)methyl)-1-((1,3-dihydroxypropan-2-yloxy)methyl)pyrimidine-2,4(1H,3H)-dione (9) Yield 3.2 g (84\%), m.p. $190-192{ }^{\circ} \mathrm{C} .{ }^{1} \mathrm{H}$ NMR (DMSO- $d_{6}$, $400 \mathrm{MHz}) \delta 3.40-3.58\left(\mathrm{~m}, 5 \mathrm{H}, 2 \mathrm{CH}_{2}, \mathrm{CH}\right), 4.53(\mathrm{~m}, 2 \mathrm{H}$, $\mathrm{OH}), 5.33$ (s, $2 \mathrm{H}, \mathrm{CH}_{2}$ phenoxy), $5.42\left(\mathrm{~s}, 2 \mathrm{H}, \mathrm{OCH}_{2} \mathrm{~N}\right)$, 5.69 (s, 1H, CH uracil), 7.31-7.64 (m, 3H, Ar-H), 12.10 (br s, $1 \mathrm{H}, \mathrm{NH}) .{ }^{13} \mathrm{C}$ NMR (DMSO- $\left.d_{6}, 100 \mathrm{MHz}\right) \delta 61.0,65.1$, 70.2, 80.6, 110.1, 115.4, 116.0, 122.8, 125.9, 128.6, 129.8, $151.9,152.0,152.2,163.2$. MS $m / z=390$ [M-1]. Anal. Calcd for $\mathrm{C}_{15} \mathrm{H}_{16} \mathrm{Cl}_{2} \mathrm{~N}_{2} \mathrm{O}_{6}$ : C, 46.05; $\mathrm{H}, 4.12 ; \mathrm{N}, 7.16$. Found: C, 46.14; H, 4.20; N, 7.09.

6-((2,4-Dichlorophenoxy) methyl)-1-(3,4-dihydroxy -5-(hydroxymethyl)tetrahydrofuran-2-yl)pyrimidine -2,4(1H,3H)-dione (16)

Yield $2.7 \mathrm{~g}$ (65\%), m.p. $128-130{ }^{\circ} \mathrm{C} .{ }^{1} \mathrm{H}$ NMR (DMSO- $d_{6}$, $400 \mathrm{MHz}) \delta 3.40-3.44$ (m, 2H, H-5',5”), 3.58-3.60 (m, 1H, H-4'), 3.70-3.85 (m, 1H, H-3'), 4.10-4.25 (m, 1H, H-2'), $4.15(\mathrm{~d}, 1 \mathrm{H}, \mathrm{OH}), 4.60(\mathrm{~d}, 1 \mathrm{H}, \mathrm{OH}), 4.90$ (s, $2 \mathrm{H}, \mathrm{CH}_{2}$ phenoxy), $5.13(\mathrm{~m}, 1 \mathrm{H}, \mathrm{OH}), 5.72(\mathrm{~s}, 1 \mathrm{H}, \mathrm{CH}$ uracil), 6.08 (d, $\left.1 \mathrm{H}, J=7.75 \mathrm{~Hz}, \mathrm{H}-1^{\prime}\right), 7.20-7.62$ (m, 3H, Ar-H), 11.91 (br s, $1 \mathrm{H}, \mathrm{NH}) .{ }^{13} \mathrm{C}$ NMR (DMSO- $\left.d_{6}, 100 \mathrm{MHz}\right) \delta 62.6,65.7$, 70.52, 71.1, 84.7, 87.7, 98.0, 115.6, 122.9, 125.8, 128.5, 129.0, 133.7, 150.6, 152.2. MS $m / z=421.1[\mathrm{M}+2]$. Anal. Calcd for $\mathrm{C}_{16} \mathrm{H}_{16} \mathrm{Cl}_{2} \mathrm{~N}_{2} \mathrm{O}_{7}$ : C, 45.84; $\mathrm{H}, 3.85 ; \mathrm{N}, 6.68$. Found: C, 45.92; H, 3.93; N, 6.76.

6-((2,4-Dichlorophenoxy)methyl)-1-(4-hydroxy-5-(hydroxymethyl)tetrahydrofuran-2-yl)pyrimidine-2,4 $(1 H, 3 H)$-dione $(18)$
Yield 2.8 g (69.7\%), m.p. $195-197^{\circ} \mathrm{C} .{ }^{1} \mathrm{H}$ NMR (DMSO- $d_{6}$, $400 \mathrm{MHz}) \delta 2.15-2.21$ (m, 2H, H-2',2”), 3.22-3.31 (m, $2 \mathrm{H}$, H-5,5”), 3.81(br s, 2H, $2 \times \mathrm{OH}), 4.50$ (m, 1H, H-3'), 4.80 (s, $2 \mathrm{H}, \mathrm{CH}_{2}$ phenoxy), $5.08(\mathrm{~m}, 1 \mathrm{H}, \mathrm{H}-4$ ') $5.40(\mathrm{~s}, 1 \mathrm{H}, \mathrm{CH}$ uracil), 5.51 (m, 1H, H-1'), 7.06-7.70 (m, 3H, Ar-H), 12.10 (br s, $1 \mathrm{H}, \mathrm{NH}) .{ }^{13} \mathrm{C}$ NMR (DMSO- $\left.d_{6}, 100 \mathrm{MHz}\right) \delta 61.7$, 65.6, 71.5, 81.1, 86.0, 87.7, 98.3, 115.6, 122.9, 125.8, 128.6, 129.9, 150.1, 151.1, 152.2, 163.1. MS $m / z=405.2[\mathrm{M}+2]$. Anal. Calcd for $\mathrm{C}_{16} \mathrm{H}_{16} \mathrm{Cl}_{2} \mathrm{~N}_{2} \mathrm{O}_{6}$ : C, 47.66; H, 4.00; N, 6.95. Found: C, 47.59; H, 4.05; N, 6.87.

6-((2,4-Dichlorophenoxy)methyl)-1-(3,4,5-trihydroxy -6-(hydroxymethyl)tetrahydro- $2 H$-pyran-2-yl)pyrimidine-2,4(1H,3H)-dione (20)

Yield 2.5 g (55.7 \%), m.p. $214-216^{\circ} \mathrm{C} .{ }^{1} \mathrm{H}$ NMR (DMSO- $d_{6}$, $400 \mathrm{MHz}) \delta 2.80-2.90$ (m, 1H, H-3'), 3.19-3.45 (m, 4H, H-4', H-5, H-6,6”), 4.10 (dd, $1 \mathrm{H}, J_{1,2}=9.95 \mathrm{~Hz}, J_{2,3}=9.15 \mathrm{~Hz}$, $\mathrm{H}-2$ '), 4.10 (m, $4 \mathrm{H}, 4 \times \mathrm{OH}), 4.71\left(\mathrm{~s}, 2 \mathrm{H}, \mathrm{CH}_{2}\right.$ phenoxy), 5.30 (d, $1 \mathrm{H}, J=9.95 \mathrm{~Hz}, \mathrm{H}-1$ '), 5.55 (s, 1H, CH uracil), 7.02-7.55 (m, $3 \mathrm{H}$, Ar-H), 8.20 (br s, $1 \mathrm{H}, \mathrm{NH}) .{ }^{13} \mathrm{C}$ NMR (DMSO- $d_{6}$, $100 \mathrm{MHz}) \delta 61.4,65.8,68.4,70.4,78.3,81.2,83.3,96.9,99.1$, $115.6,122.8,125.7,128.6,129.8,152.2,163.7,166.5$. MS m/z $=451.1[\mathrm{M}+2]$. Anal. Calcd for $\mathrm{C}_{17} \mathrm{H}_{18} \mathrm{Cl}_{2} \mathrm{~N}_{2} \mathrm{O}_{8}: \mathrm{C}, 45.45 ; \mathrm{H}$, 4.04; N, 6.24. Found: C, 45.51; H, 4.09; N, 6.17.

\section{Results and Discussion}

Our strategy to design and synthesize pyrimidine nucleoside analogues was based on the alkylation of si-<smiles>[R]Cc1cc(=O)[nH]c(=O)[nH]1</smiles>

Scheme 1. Synthesis of acyclic nucleoside analogues containing pyrimidine moiety 6-11 
lylated pyrimidine following Vorbrüggen and Niedballa's procedure. ${ }^{33-35}$ The intermediate, bis(trimethylsilyl) 2 , was prepared by the silylation of 6-(2,4-dichlorophenoxymethyl)pyrimidine-2,4-dione (1) using hexamethyldisilazane (HMDS) and subjected to react with different acyclic sugar analogues, namely (2-acetoxyethoxy)methyl acetate (3), 2-(acetoxymethoxy)propane-1,3-diyl dibenzoate (4) and benzyloxymethyl acetate (5) to afford the corresponding protected nucleosides analogues $\mathbf{6 , 8}$ and 10, 11, respectively (Scheme 1).

Structures of new acyclic nucleoside analogues were fully characterized by analytical and spectral methods $\left({ }^{1} \mathrm{H}\right.$ NMR, ${ }^{13} \mathrm{C}$ NMR, and elemental analysis). The ${ }^{1} \mathrm{H}$ NMR showed the disappearance of $-\mathrm{NH}$ proton which was detected in the parent compound $\mathbf{1}$ and instead a new signal appeared at the range $\delta 5.34-5.62$ ppm characteristic for ($\mathrm{OCH}_{2} \mathrm{~N}$ ) indicative for the acyclic nucleosides formation. It is interesting to note that the reaction of 2 with benzyloxymethyl acetate (5) afforded two products 10 and $11 .{ }^{1} \mathrm{H}$ NMR showed the disappearance of the two - NH protons of 1 and two new signals appeared at 5.34 and 5.46 ppm at-

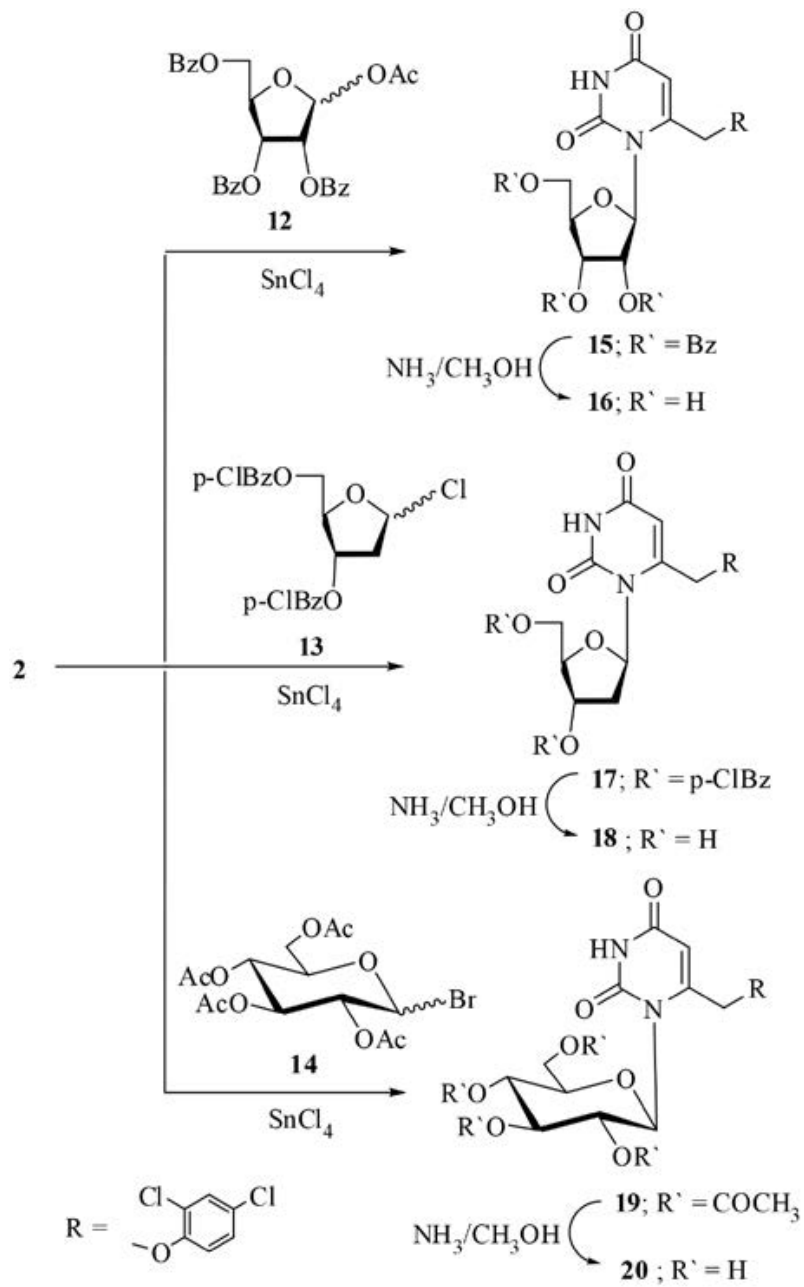

Scheme 1. Synthesis of acyclic nucleoside analogues containing pyrimidine moiety 6-11 tributed to $\left(-\mathrm{OCH}_{2} \mathrm{~N}^{1}\right.$ and $\left.-\mathrm{OCH}_{2} \mathrm{~N}^{3}\right)$, supporting the formation of $\mathbf{1 1}$ via double alkylation of $\mathbf{2}$ (cf. Experimental).

Deprotection of compounds $\mathbf{6}$ and $\mathbf{8}$ was achieved by cleavage of the ester blocking group with methanolic ammonia solution to give compounds $\mathbf{7}$ and $\mathbf{9}$ in fair to moderate yield, respectively (c.f. Experimental). In the continuation of our study, compound $\mathbf{1}$ was reacted with various activated cyclic sugars, namely 2-acetoxy-5-(benzoyloxymethyl)tetrahydrofuran-3,4-diyl dibenzoate (12), 5-chloro-2-((4-chlorobenzoyloxy)methyl)tetrahydrofuran-3-yl 4-chlorobenzoate (13) and 2-(acetoxymethyl)-6-bromotetrahydro- $2 \mathrm{H}$-pyran-3,4,5-triyl triacetate (14) under the same procedure as previously, giving the protected nucleosides 15,17 and 19 as $\beta$-anomers. ${ }^{1} \mathrm{H}$ NMR showed a doublet signal at $\delta 6.20-6.51 \mathrm{ppm}$ corresponding to the anomeric proton of the sugar moiety with the spin-spin coupling constant $\left(J_{1,2}=9.10-9.50 \mathrm{~Hz}\right)$ which can be attributed to the diaxial orientation of $\mathrm{H}-1$ and $\mathrm{H}-2$ protons indicating the presence of $\beta$-configuration. Compounds 15, 17, and 19 were deprotected by using methanolic ammonia solution at room temperature to give compounds $\mathbf{1 6}, \mathbf{1 8}$, and $\mathbf{2 0}$, respectively (Scheme 2).

\section{Conclusions}

A series of cyclic and acyclic nucleosides were prepared with moderate yields by alkylation of 6-(2,4-dichlorophenoxymethyl)pyrimidine-2,4-dione with various acyclic and cyclic activated sugars by performing Vorbrüggen and Niedballa's procedure. Deprotection of the synthesized nucleosides was achieved by using methanolic ammonia solution.

\section{Acknowledgments}

The authors acknowledge Professor Chris Meier and Innovative Research Team in State Key laboratory of Organic Chemistry, Hamburg University, for their help during performing this work.

\section{References}

1. E. De Clercq, A. Holý, Nat. Rev. Drug Discovery. 2005, 4, $928-$ 240. DOI: $10.1038 / \mathrm{nrd} 1877$

2. D. F. Ewing, V. Glaçon, G. Mackenzie, C. Len, Tetrahedron Lett. 2002, 43, 989-991. DOI:10.1016/S0040-4039(01)02298-5

3. D. Pansuriya, K. Menpara, N. Kachhadiya, J. Menpara, K. Ladva, J. Appl. Chem. 2014, 3, 2335-2342.

4. R. C. Elderfield, Heterocyclic compounds, John Wiley \& Sons, Inc., New York, 1957, Vol. 6, p 744.

DOI:10.1002/jps.3030460623

5. A. E. Porter, Diazines and benzodiazines (Netherlands: Pregamon Press, Elsevier Science) BV 6. Amsterdam, 1979, p.14. 
6. C. O. Kappe, Tetrahedron. 1993, 49, 6937-7168. DOI:10.1016/S0040-4020(01)87971-07

7. A. L. S. Rodrigues, J. M. Rosa, V. M. Gadotti, Pharmacol., Biochem. Behav. 2005, 82, 156-162.

DOI:10.1016/j.pbb.2005.08.003

8. J. S. Bradley, R. Guidos, S. Baragona, Lancet Infect. Dis. 2007, 7, 68-78. DOI:10.1016/S1473-3099(06)70689-2

9. N. M. Thumar, A. A. Kaneria, M. Vadodaria, K. Ladva, Int. Lett. Chem. Phys. Astron. 2016, 65, 11-26.

DOI:10.18052/www.scipress.com/ILCPA.65.11

10. H. P. Schweizer, Microbiology Lett. 2001, 202, 1-7. DOI:10.1111/j.1574-6968.2001.tb10772.x

11. K. Poole, J. App. Microbiol. 2002, 92, 55-64S. DOI:10.1046/j.1365-2672.92.5s1.8.x

12. K. Poole, J. Pharm. Pharmacol. 2001, 53, 283-294. DOI:10.1211/0022357011775514

13. P. W. Taylor, P. D. Stapleton, J. P. Luzio, Drug Discov. Today. 2002, 7, 1086-1091. DOI:10.1016/S1359-6446(02)02498-4

14. Y. Ju, D. Kumar, R. S. Varma, J. Org. Chem. 2006, 71, 66976700. DOI:10.1021/jo061114h

15. M. F. El Shehry, J. Balzarini, C. Meier, Synthesis. 2009, 5, 841847. DOI:10.1055/s-0028-1083369

16. Y. Ju, R. S. Varma, J. Org. Chem. 2006, 71, 135-141. DOI:10.1021/jo051878h

17. C. A. Zificsak, D. J. Hlasta, Tetrahedron. 2004, 60, 8991-9016. DOI:10.1016/j.tet.2004.07.016

18. T. Haino, M. Tanaka, K. Ideta, K. Kubo, A. Mori, Y. Fukazawa, Tetrahedron Lett. 2004, 45, 2277-2279.

DOI:10.1016/j.tetlet.2004.01.116

19. A. Manlove, M. P. Groziak, J. Heterocycl. Chem. 2009, 21, 375-414. DOI:10.1016/S0959-6380 (9)70040-6

20. R. Singh, A. Chouhan, World J. Pharm. Pharm. Sci. 2014, 3, 574-597.

21. M. Hassan, D. van der Lelie, D. Springael, U. Römling, N. Ahmed, M. Mergeay, Gene. 1999, 238, 417-425.

DOI:10.1016/S0378-1119(99)00349-2
22. D. M. Livermore, Intensive Care Med. 2000, 26, S14-21. DOI:10.1007/s001340051113

23. L. Jeu, F. J. Piacenti, A. G. Lyakhovetskiy, H. B. Fung, Voriconazole. Clin. Ther. 2003, 25, 1321-1381.

DOI:10.1016/S0149-2918(03)80126-1

24. P. D. Lokhande, B.Y. Waghamare, S. S. Sakate, Indian J. Chem. Sect. B. 2005, 44, 2338-2342.

25. G. J. Reddy, D. Manjula, K. S. Rao, M. Khalilullah, D. Latha, Indian J. Chem. Sect. B. 2005, 44, 2412-2415.

26. M. García-Valverde, T. Torroba, Molecules. 2005, 10, 318-320. DOI: $10.3390 / 10020318$

27. D. W. Hopper, A. L. Crombie, J. J. Clemens, S. Kwon, Prog. Heterocycl. Chem. 2009, 21, 330-374.

DOI:10.1016/j.pbb.2005.08.003

28. J. Tani, Y. Yamada, T. Oine, T. Ochiai, R. Ishida, I. Inoue, J. Med. Chem. 1979, 22, 95-99. DOI:10.1021/jm00187a021

29. B. Kumar, B. Kaur, J. Kaur, A. Parmar, R. D. Anand, H. Kumar, Indian J. Chem. Sect. B. 2002, 41, 1526-1530.

DOI:10.1155/2014/202784

30. S. V. Dinakaran, B. Bomma, K. K. Spinivasan, Pharm. Chem. 2012, 4, 255-265.

31. M. A. Abu-Zaied, E. M. El-Telbani, G. H. Elgemeie, G. A. M. Nawwar, Eur. J. Med. Chem. 2011, 46, 229-235.

DOI:10.1016/j.ejmech.2010.11.008

32. M. F. El Shehry, R. H. Swellem, S. M. Abu-Bakr, E. M. El-Telbani, Eur. J. Med. Chem. 2010, 45, 4783-4787.

DOI:10.1016/j.ejmech.2010.07.043

33. E. M. El-Telbani, S. M. Ghanem, F. A. El Essawy, A. F. ElSayed, Lett. Drug. Des. Discov. 2011, 8, 822-829.

DOI:10.2174/1570180811108090822

34. U. Niedballa, H. Vorbrüggen, J. Org. Chem. 1974, 39, 36543660. DOI:10.1021/jo00939a008

35. U. Niedballa, H. Vorbrüggen, J. Org. Chem. 1976, 41, $2084-$ 2086. DOI:10.1021/jo00874a002

36. H. Vorbrüggen, K. Krolikiewiecz, B. Bennua, Chem. Ber. 1981, 114, 1234-1255. DOI:10.1002/cber.19811140404

\section{Povzetek}

$\mathrm{V}$ članku predstavljamo priročno metodo za pripravo cikličnih in acikličnih nukleozidov s pomočjo alkiliranja 6-(2,4-diklorofenoksimetil)pirimidin-2,4-diona (1) s širokim izborom acikličnih in cikličnih aktiviranih analogov sladkorjev: (2-acetoksi)metil acetat (3), 2-(acetoksimetoksi)propan-1,3-diil dibenzoat (4), benziloksimetil acetat (5), 2-acetoksi-5-(benzoiloksimetil)tetrahidrofuran-3,4-diil dibenzoat (12), 5-kloro-2-((4-klorobenzoiloksi)metil)tetrahidrofuran-3-il 4-klorobenzoat (13) in 2-(acetoksimetil)-6-bromotetrahidro-2H-piran-3,4,5-triil triacetat (14). Odstranitev zaščitne skupine iz tako pripravljenih nukleozidov smo dosegli z uporabo amonijaka v metanolu. Nove nukleozide, ki smo jih na ta načini sintetizirali, smo tudi karakterizirali $\mathrm{z}$ analitskimi metodami (masna spektrometrija, ${ }^{1} \mathrm{H} \mathrm{NMR},{ }^{13} \mathrm{C}$ NMR in elementna analiza). 